\title{
Endothelial relaxation is disturbed by oxidative stress in the diabetic rat heart: influence of tocopherol as antioxidant
}

\author{
P. Rösen ${ }^{1}$, T. Ballhausen ${ }^{1}$, W. Bloch ${ }^{2}$, K. Addicks ${ }^{2}$ \\ ${ }^{1}$ Department of Clinical Biochemistry, Diabetes Research Institute, Düsseldorf, Germany \\ ${ }^{2}$ Anatomical Institute of the University, Cologne, Germany
}

Summary Increased oxidative stress has been suggested to contribute to disturbances in the regulation of coronary flow and the increased cardiac risk in diabetes mellitus. Using the isolated perfused heart of streptozotocin-diabetic rats our study shows that basal and maximal coronary flow (tested by infusion of sodium nitroprusside) are not altered in diabetes, but that 5-hydroxytryptamine (5-HT) stimulated endothelium-dependent increase in coronary flow becomes progressively impaired. This defect of the endothelium-dependent vasodilatation was prevented by perfusion of the hearts with superoxide dismutase and pretreatment of the diabetic rats with tocopherol-acetate. Morphological studies also revealed that pretreatment with tocopherol-acetate was cardioprotective, and largely prevented severe alterations of myocardial structure typically observed after a diabetes duration of 3 months; deterioration and fragmentation of myofilament bundles were seen less, and the numbers of areas of focal necrosis and of contraction bands were clearly reduced. In con- trast to untreated diabetic hearts the autonomic nerve fibers detected by catecholamine fluorescence were running in parallel in hearts of tocopherol-treated diabetic rats, and the amount of catecholamines was not different from that of healthy control rats. Trichrome staining and immunohistochemical staining of collagen I and III showed a dramatic increase in the number and the size of deposits of collagen fibers at precapillary locations in the diabetic hearts which were significantly reduced by anti-oxidative treatment. These findings demonstrate that oxidative stress may not only play a major role in the impairment of endothelium-dependent regulation of coronary flow, but also in the development of perivascular fibrosis and severe changes of the autonomic nerves and contractile system in myocardium. [Diabetologia (1995) 38: 1157-1168]

Key words Diabetes mellitus, myocardium, streptozotocin diabetes, vitamin $\mathrm{E}$, endothelium, coronary vessels, autonomic neuropathy.
In numerous clinical and epidemiological studies evidence has been presented that human diabetic patients are particularly susceptible to heart failure [1$3]$. This high cardiac risk in diabetes is not necessar-

Received: 25 February 1995 and in revised form: 15 May 1995

Corresponding author: Professor P. Rösen, Diabetes Research Institute, Auf'm Hennekamp 65, D-40225 Düsseldorf, Germany

Abbreviations: 5-HT, 5-Hydroxytryptamine; NO, nitric oxide; EDRF, endothelial-dependent relaxation factor; NP, sodium nitroprusside; L-NAME, nitro-L-arginine-mono-methyl-ester; SOD, superoxide dismutase. ily associated with coronary atherosclerosis, since it has also been observed in a significant number of young patients who do not show any symptoms of coronary heart disease [3]. This conclusion is also supported by experimental studies using animal models in which diabetes was induced chemically or developed spontaneously as in $\mathrm{BB}$ rats [4-7]. It has been postulated that wall thickening and luminal narrowing of the intramural coronary microvasculature $[3,8]$ reduce the coronary flow reserve $[3,8]$ and limit the adaptation of coronary flow to the metabolic demand.

It has become clear in recent years that the endothelium has a large impact on the regulation of vaso- 
tonus of the peripheral and coronary vasculature by the release of specific mediators such as prostaglandins, endothelium-derived relaxing and contracting factors, endothelins or angiotensin II $[9,10]$. Disturbances of endothelium-dependent regulation of vascular flow have not only been demonstrated in disease states such as atherosclerosis [11], hypercholesterolaemia [12], and hypertension [13], but also in diabetes $[14,15]$.

The underlying mechanisms have not yet been established, but it has been suggested that the generation of endothelium-dependent relaxation factor (EDRF) which has now been identified as nitric oxide (NO) or an NO-substituted compound, is diminished in diabetes $[14,16-19]$. Furthermore, the biological availability of NO might be reduced in diabetic states, since it is rapidly quenched by reactive oxygen radicals or by advanced glycation endproducts $[20,21]$. Alternatively, the diminished endothelium-dependent vasodilatation might be caused by the generation of vasoconstricting prostaglandin-endoperoxides counteracting the dilating effect of $\mathrm{NO}$ [22-24].

Since endothelium-dependent dilatation has mostly been studied in large vessels such as the aorta, it is unclear at this time whether diabetes affects the smaller vessels, in particular the resistance vessels, in a similar way. Furthermore, endothelium shows widely different properties depending on the location in the vascular tree. Therefore, it is not yet known in which way diabetes specifically affects the endothelium-dependent regulation of coronary flow. A recent study by Nitenberg et al. [25] suggests that the endothelium-dependent relaxation of coronary arteries is also impaired in both insulin-dependent and non-insulin-dependent diabetic patients, but this study, again, mainly determined the reactivity of the large conductive coronaries.

To gain a deeper insight into the mechanisms by which diabetes affects the endothelium-dependent regulation of vascular flow in the resistance vessels of the heart, we studied the endothelium-dependent regulation of coronary flow in the isolated rat heart, which contains an intact perfusion system and consists mainly of capillaries and small arterioles which determine coronary resistance. To study whether the endothelial-dependent relaxation by NO is affected by diabetes, rat hearts were perfused with 5 -hydroxytryptamine $(5-\mathrm{HT})$ to stimulate the release of $\mathrm{NO}$. We here present evidence that enhanced oxidative stress is responsible for the impairment of endothelium-dependent vasodilatation in the diabetic heart, and can be prevented by treatment of the animals with high doses of tocopherol-acetate.

On the other hand, NO is not only a potent vasodilating compound, but is also involved in the regulation of catecholamine release from sympathetic nerves [26]. Inhibition of NO synthesis has been shown to lead to an activation of the sympathetic nervous system [26], which is a frequent complication in human and experimental diabetes [27]. It has been suggested, furthermore, that in diabetes an activation of the sympathetic nervous system contributes to myocardial damage either by the generation of cytotoxic free radicals derived from catecholamines [28] or by induction of hypertrophy and growth factors resulting in an increased expression of collagen in myocardium $[27,29,30]$. Prevention of NO inactivation by oxidative stress is therefore expected to protect the heart in diabetes by at least two different mechanisms: directly, by restoring the vasodilatory effect of NO and indirectly, by prevention of a cascade of events associated with the excessive activation of the sympathetic nervous system. Analysis of the effect of treatment of diabetic rats with tocopherol on myocardial structure, catecholamines and collagen was performed in order to examine this hypothesis.

\section{Materials and methods}

Animals. Male Wistar rats (250-290 g, Winkelmann, Borchen, Germany) had free access to water and diet and were housed under standard conditions in the animal laboratory of the Diabetes Research Institute. Diabetes was induced by intraperitoneal application of streptozotocin $(60 \mathrm{mg} / \mathrm{kg}$ body weight) as previously described [5,31]. Manifestation of diabetes was verified 1 week after injection of streptozotocin by determination of blood glucose (Hexokinase method), glucosuria (HaemoGlukotest, Boehringer, Mannheim, Germany) and glycated haemoglobin (Isolab, Munich, Germany). Only animals with a blood glucose level higher than $15 \mathrm{mmol} / \mathrm{l}$ and an $\mathrm{HbA}_{1}$ level higher than $12 \%$ were considered to be diabetic.

Treatment. Control animals received a standard laboratory chow for rats (Sniff, Soest, Germany) containing $163 \mathrm{mg}$ tocopherol equivalents $/ \mathrm{kg}$ diet. Additionally, groups of animals received vitamin E-supplemented or -deficient diets. To obtain a high-vitamin $\mathrm{E}$ diet, the control diet was supplemented with tocopherol acetate (alpha-tocopherol-acetate as Corvitol, Henkel AG, Düsseldorf, Germany) to increase the amount of vitamin $\mathrm{E}$ to $1.3 \mathrm{~g}$ tocopherol acetate $/ \mathrm{kg}$ corresponding to 610 tocopherol equivalents $/ \mathrm{kg}$ diet. The vitamin E-deficient diet contained $6 \mathrm{mg}$ tocopherol equivalents $/ \mathrm{kg}$ chow. The diabetes duration is shown in Tables 1-3.

Heart perfusion. Isovolumic, spontaneously beating hearts were perfused according to Langendorff as described previously $[5,31]$. The perfusion pressure was kept constant at $70 \mathrm{~mm} \mathrm{Hg}$. The modified "Krebs-Henseleit" perfusion buffer contained (in mmol/l): $118 \mathrm{NaCl}, 4.8 \mathrm{KCl}, 1.2 \mathrm{KH}_{2} \mathrm{PO}_{4}, 20 \mathrm{Na}$ $\mathrm{HCO}_{3}, 1.2 \mathrm{MgSO}_{4}, 2.0 \mathrm{CaCl}_{2}$ and 5.6 glucose. The filtered buffer was equilibrated at $37^{\circ} \mathrm{C}$ with carbogen gas $\left(95 \% \mathrm{O}_{2}\right.$, $5 \% \mathrm{CO}_{2}, \mathrm{pH} 7.4$ ).

Preparation. Animals were anaesthetized by ether. After thoracotomy the hearts were immediately connected to a non-circulating perfusion system (at $37^{\circ} \mathrm{C}$ ). Venae cavae were ligated and the hearts were carefully excised from the thorax. A latex balloon was placed into the left ventricle via the opened left at- 


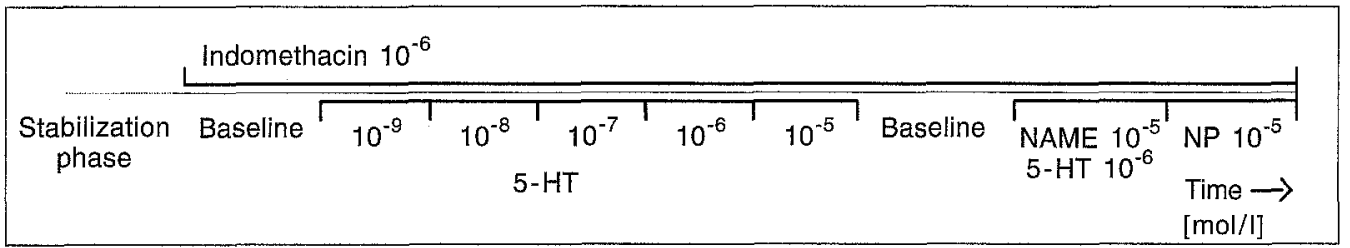

Fig.1. Perfusion schedule. Rat hearts isolated and perfused as described in Methods. After an equilibration period basal flow was measured; then the dose-efficacy of 5-HT stimulated coronary flow was recorded, followed by perfusion with $\mathrm{L}$ NAME to inhibit the release of NO. At the end of perfusion

rium and connected to a pressure transducer. Left ventricular peak systolic pressure and heart rate were simultaneously registered. Coronary flow was measured continuously by calibrated glass cylinders.

Experimental protocol (Fig. 1). After preparation, there was a 25-min stabilization phase to ensure constant mechanical and flow properties. To measure endothelial-dependent relaxation hearts were perfused with increasing concentrations of 5-HT $(1 \mathrm{nmol} / \mathrm{l}-10 \mu \mathrm{mol} / \mathrm{I})$. The increase in coronary flow was measured and the cumulative dose-efficacy curves determined. Thereafter, the NO formation was inhibited by perfusing the heart with nitro-L-arginine-mono-methyl-ester (L-NAME; $10 \mu \mathrm{mol} / \mathrm{l})$ to verify that the observed increase in coronary flow was specifically induced by NO. Maximal coronary flow, was induced independently from endothelium by direct application of sodium nitroprusside (NP; $10 \mu \mathrm{mol} / 1)$ in the perfusion fluid. NP and the various concentrations of 5-HT were infused for at least $10 \mathrm{~min}$ to obtain a constant coronary flow rate. All experiments were performed in the presence of indomethacin ( $3 \mu \mathrm{mol} / 1)$ to inhibit the cyclooxygenase activities and eliminate the generation of interfering prostaglandins.

Where indicated, the hearts were perfused with superoxide dismutase (SOD, $50 \mathrm{U} / \mathrm{mg}$, Boehringer, Mannheim, Germany) at a concentration of $50 \mu \mathrm{U} / \mathrm{ml}$.

\section{Morphology}

Preparation of the rat heart. Rat hearts were retrogradely perfused according to the Langendorff technique. Hearts were perfused at a constant pressure of $60 \mathrm{~cm} \mathrm{H}_{2} \mathrm{O}$ with a modified Krebs Henseleit buffer (in mmol/l): $1.8 \mathrm{CaCl}_{2}, 1.05 \mathrm{MgCl}_{2}$, $5.35 \mathrm{KCl}, 136.9 \mathrm{NaCl}, 0.42 \mathrm{NaH}_{2} \mathrm{PO}_{4}, 10.1$ glucose, $23.8 \mathrm{Na}-$ $\mathrm{HCO}_{3}$ ) pre-warmed to $37^{\circ} \mathrm{C}$ and equilibrated with $95 \% \mathrm{O}_{2} /$ $5 \% \mathrm{CO}_{2}$. Following the pressure constant perfusion with the modified Krebs-Henseleit solution, the hearts were perfused with a $0.1 \mathrm{~mol} / \mathrm{l}$ cacodylate buffered $2 \%$ glutaraldehyde $2 \%$ paraformaldehyde fixative via the cannula located within the aorta near the coronary ostia.

Light-and electron-microscopical preparation. Left papillary muscles were replaced and further fixed in $0.1 \mathrm{~mol} / 1$ cacodylate buffered $2 \%$ glutaraldehyde $/ 2 \%$ paraformaldehyde followed by postfixation in $2 \%$ osmium tetroxide buffered at $\mathrm{pH}$ 7.3 with $0.1 \mathrm{~mol} / 1$ sodium cacodylate for $2 \mathrm{~h}$ at $40^{\circ} \mathrm{C}$. The specimens were rinsed in cacodylate buffer three times, block stained in $1 \%$ uranyl acetate in $70 \%$ ethanol for $8 \mathrm{~h}$, dehydrated in a series of graded ethanol and embedded in araldite. Semithin sections of plastic embedded papillary muscles were stained with methylene blue and investigated with the aid of a computerized morphometrical analysis-unit (Leica CBA8000, maximal coronary flow capacity was determined by perfusion with NP $(10 \mu \mathrm{mol} / 1)$. To inhibit the release of interfering prostaglandins heart perfusion was performed in the presence of indomethacin $(3 \mu \mathrm{mol} / 1)$

Köln, Germany). Ultrathin sections (30-60 nm) were obtained with a diamond knife on a Reichert ultramicrotome (Nussloch, Germany), placed on copper grids, and examined with a Zeiss EM 902A electron microscope (Oberkochen, Germany). For light microscopical observations ten semithin sections, and for electronmicroscopy five thin sections of the left papillary muscle were studied, per heart.

Catecholamine fluorescence. The right ventricles of isolated perfused hearts were rapidly cut off, freeze-clamped with a Wollenberger clamp cooled in liquid nitrogen and stored at $-80^{\circ} \mathrm{C}$ for histochemical examination of intra-axonal catecholamine stores. At a temperature of $-30^{\circ} \mathrm{C}$, the frozen myocardial tissue consisted of 16.5-um cryostat serial sections. Adrenergic nerve fibers of the right ventricle were made visible by means of glyoxylic acid-induced fluorescence of intraneuronal catecholamines using the method of De La Torre and Surgeon [32]. Quantitative assessment of individual tissue sections was performed by high resolution microfluorimetry. The system consisted of a Leitz orthoplan microscope equipped for fluorescence with epi-illumination and $3 \mathrm{~mm} B G 12$ and Leitz $\mathrm{K}$ 490 primary and secondary filters (Köln, Germany), respectively. Using a residual light-amplifying caesicon camera, tissues under study were focussed in such a way that within the visible field of the individual preparation, only sections with nerve fibers running in parallel were processed and sections containing sympathetic plexuses were excluded from measurement. After inversion of the primary image by means of a computerized image analysing system (Artek 982, Bio-Sys, Karben, Germany), fluorescing adrenergic nerves were easily detectable as dark areas against a bright background. To eliminate background fluorescence, points below a given threshold intensity were filled up in brightness with the help of a specially-designed measuring mask so that only fluorescing nerve fibers remained visible. For determination of the fluorescing area one section per heart was taken from the right ventricle at a distance of $150 \mu \mathrm{m}$ from the epicardial surface. The total observation area was $3 \mathrm{~mm}^{2}$ per heart. The amount of the fluorescing area was estimated from 100 consecutive measurements per section and is given as a percentage of the total area. No correction for fading of the fluorescent image by photodecomposition was necessary, as the whole process was performed in $10 \mathrm{~s}$. By using this technique not only the length and thickness of the adrenergic nerve fibers, but also the dimensions of their axonal dilatation (the varicosities) were recorded.

Trichrome technique. For trichrome staining 1-mm blocks of myocardium containing parts of the left coronary artery were embedded in paraffin and 5 - $\mu \mathrm{m}$-thick slices were sectioned transversally to the longitudinal axis of the arteries. The slices were stained using a standard method described by Goldener [33] to distinguish between connective tissue from myocytes, 
Table 1. Influence of streptozotocin-diabetes on heart and body weight

\begin{tabular}{lllll}
\hline Group & $\begin{array}{l}\text { Age } \\
\text { (weeks) }\end{array}$ & $\begin{array}{l}\text { Diabetes } \\
\text { (weeks) }\end{array}$ & $\begin{array}{l}\text { Body weight } \\
(\mathrm{g})\end{array}$ & $\begin{array}{l}\text { Heart weight } \\
\text { (g dry weight) }\end{array}$ \\
\hline A: Control & 15 & - & $363 \pm 17$ & $0.183 \pm 0.006$ \\
B: Control & 26 & - & $413 \pm 19^{\mathrm{a}}$ & $0.221 \pm 0.008^{\mathrm{a}}$ \\
C: Diabetes & 15 & 5 & $338 \pm 18$ & $0.180 \pm 0.010$ \\
D: Diabetes & 26 & 17 & $330 \pm 10^{\mathrm{b}}$ & $0.175 \pm 0.003^{\mathrm{b}}$ \\
\hline
\end{tabular}

Data are mean $\pm \operatorname{SEM}(n=5-8$ animals)

${ }^{\mathrm{a}} p<0.05$ group A vs group B

${ }^{\mathrm{b}} p<0.05$ group $\mathrm{B}$ vs group $\mathrm{D}$

smooth muscle and endothelial cells by different colouring. The areas of connective tissue and the perimeter of coronary vessels were automatically detected and measured on a Leitz Medilux microscope connected with a Leitz CBA8000 image analysing system for detection of real colour. The area of connective tissue is given by the trichrome stained area (c in $\mu \mathrm{m}^{2}$ ) related to the circumference of the vessel $(\mathrm{v}$ in $\mu \mathrm{m})$ as $\mathrm{c} / \mathrm{v}$ $\left(\mu \mathrm{m}^{2} / \mu \mathrm{m}\right)$. For each heart, slices from five different parts of the left coronary artery were selected. The distance between the slices was set at more than $1 \mathrm{~mm}$.

Immunohistochemical staining for collagen I and III. Slices of 5 - $\mu \mathrm{m}$ thickness, embedded in paraffin, from five different parts of the left coronary artery of each heart were selected and measured as described above. The slices were de-paraffinized and pretreated with $0.1 \%$ trypsin (Sigma Chemical Co., Deisenhofen, Germany) for $10 \mathrm{~min}$ at $37^{\circ} \mathrm{C}$. Following several washing steps (phosphate buffered saline) the sections were incubated for $2 \mathrm{~h}$ at $37^{\circ} \mathrm{C}$ with the primary antibodies: rabbit anti-rat collagen I and collagen III, respectively (Biermann, Bad Nauheim, Germany). The antigen-antibody complex was then made visible by a combination of donkey anti-rabbit biotin conjugated secondary antibody (Dianova, Hamburg, Germany) and a peroxidase complex (Amersham, Braunschweig, Germany). As chromogen we used diaminobenzidine (Dako, Hamburg, Germany).

Reagents. Indomethacin was dissolved in $100 \mathrm{mmol} / 1$ Tris- $\mathrm{HCl}$ ( $\mathrm{pH} 8.7), 5-\mathrm{HT}, \mathrm{L}-\mathrm{NAME}$ and NP were dissolved in distilled water. For perfusion the drugs were further diluted with the perfusion buffer.

Tocopherol. Tocopherol in plasma was determined by high pressure liquid chromatography as described by Diplock [34].

Data analysis. Coronary flow rates were corrected for heart weight and are given as $\mathrm{ml} / \mathrm{g}$ dry weight. Alternatively, coronary flow rates are related to the maximal coronary flow observed for each heart and are given as a percentage of the maximal flow determined after application of NP. The concentra- tions of 5-HT inducing half-maximal increase of coronary flow $\left(\mathrm{EC}_{50}\right)$ were calculated by curve fitting ('InPlot 4.0 ' GraphPad Software).

\section{Statistical analysis}

Statistical analysis was performed by Wilcoxon's test, $p$ values less than 0.05 (two-tailed) were considered to be significantly different. Results are presented as the mean \pm SEM of $n$ individual experiments. Statistical analysis was performed using the BMDP-PC 90 statistical program. Differences between group means were determined by calculation of one-way analysis of variance (ANOVA). Multiple comparisons were performed using adjusted $t$-tests with $p$ values corrected by the Bonferroni method.

\section{Results}

As diabetic animals, only those with blood glucose levels higher than $15 \mathrm{mmol} / \mathrm{l}$ were used. Furthermore, evidence for chronic hyperglycaemia follows from the elevated haemoglobin $\mathrm{A}_{1}(>12 \%)$ and the glucosuria generally observed in all the diabetic animals. After a diabetes duration of 5 weeks body and heart weights were not significantly different in control and diabetic rats. Only after a diabetes duration of 17 weeks or longer were the body and heart weights of diabetic rats diminished as compared to controls (Table 1).

Heart performance was similarly changed in both groups as described previously $[4,5,31]$; the maximal rates of contraction and relaxation were diminished, the maximal left ventricular systolic pressure and heart rate slightly reduced.

The spontaneous coronary flow was not significantly different in hearts of control and diabetic rats independently of the duration of diabetes. Similarly, the maximal coronary flow capacity after infusion of NP did not significantly differ between control and diabetic rats (Table 2) and was independent from the perfusion time (data not shown).

To induce endothelium-dependent relaxation of coronary vessels, hearts were perfused with 5-HT. Interference with the release of prostanoids was avoided by infusion of indomethacin during the total experiment. 5-HT caused a concentration-dependent

Table 2. Coronary flow rates $\left(\mathrm{ml} \cdot \mathrm{min}^{-1} \cdot \mathrm{g}^{-1}\right.$ dry weight) dependent on age and diabetes

\begin{tabular}{lllll}
\hline Group & $\begin{array}{l}\text { Age } \\
\text { (weeks) }\end{array}$ & $\begin{array}{l}\text { Diabetes } \\
\text { (weeks) }\end{array}$ & $\begin{array}{l}\text { Basal } \\
\left(\mathrm{ml} \cdot \mathrm{min}^{-1} \cdot \mathrm{g}^{-1} \text { dry weight) }\right.\end{array}$ & $\begin{array}{l}\mathrm{MCF} \\
\left(\mathrm{ml}^{\circ} \mathrm{min}^{-1} \cdot \mathrm{g}^{-1} \mathrm{dry} \mathrm{weight}\right)\end{array}$ \\
\hline A: Control & 15 & - & $60 \pm 5$ & $113 \pm 6^{\mathrm{a}}$ \\
B: Control & 26 & - & $55 \pm 6$ & $113 \pm 14^{\mathrm{a}}$ \\
C: Diabetes & 15 & 5 & $56 \pm 6$ & $129 \pm 9^{\mathrm{a}}$ \\
D: Diabetes & 26 & 17 & $53 \pm 3$ & $125 \pm 5^{\mathrm{a}}$ \\
\hline
\end{tabular}

Data are mean \pm SEM ( $n=5-8$ animals)

${ }^{\mathrm{a}} p<0.05$ basal vs stimulated coronary flow (MCF) 


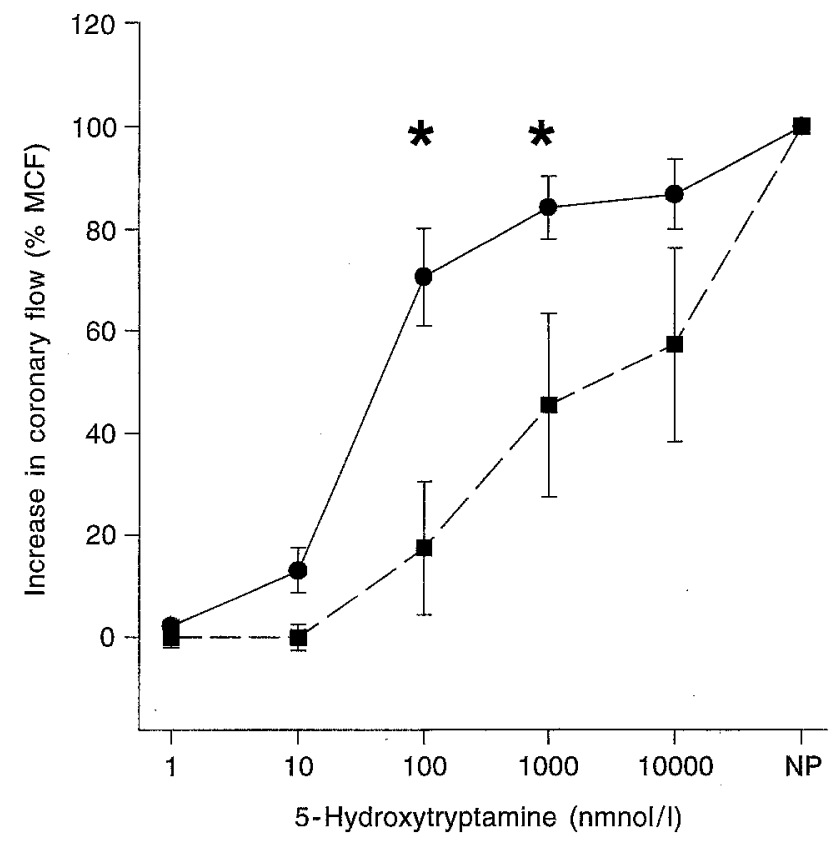

Fig. 2. Stimulation - of coronary $\square$ flow by 5-HT. Coronary flow in hearts of control and diabetic rats determined depending on the concentration of 5-HT (cumulative dose-efficacy curve). Diabetes duration was 17. Data are given as mean \pm SEM of six independent experiments. Significant differences between the various groups $* p<0.05$

increase in coronary flow in hearts of both control and diabetic rats (Fig. 2). Whereas in hearts of control rats the half-maximal concentration of 5-HT needed to increase the coronary flow was independent of the age of the rats between 15 and 26 weeks, the sensitivity of hearts of diabetic rats was progressively diminished depending on the duration of diabetes (Fig. 3). The differences in the half-maximal concentration of vasodilatation by 5 -HT between control and diabetic rats became significant after a diabetes duration of 17 and 26 weeks. If the duration of diabetes was, however, extended to more than 1 year, 5-HT did not increase the coronary flow further, and a reduction of coronary flow was observed (data not shown). In hearts of control and diabetic rats vasodilatation by 5-HT was completely inhibited by perfusion with L-NAME indicating that in both cases the relaxation of coronary vessels by 5 -HT was mediated by the release of NO.

Furthermore, we did not observe any difference in the basal coronary flow between hearts of diabetic and control rats. Perfusion with L-NAME caused a comparable reduction in coronary flow in both types of hearts indicating that the basal release of NO was not changed by diabetes lasting from 5 to 26 weeks.

Influence of superoxide dismutase. To test if the impairment of the endothelium-dependent vasodilatation is mediated by superoxide anions as suggested by various authors $[20,22]$ the influence of $5-\mathrm{HT}$ on the coronary flow was determined in the presence of

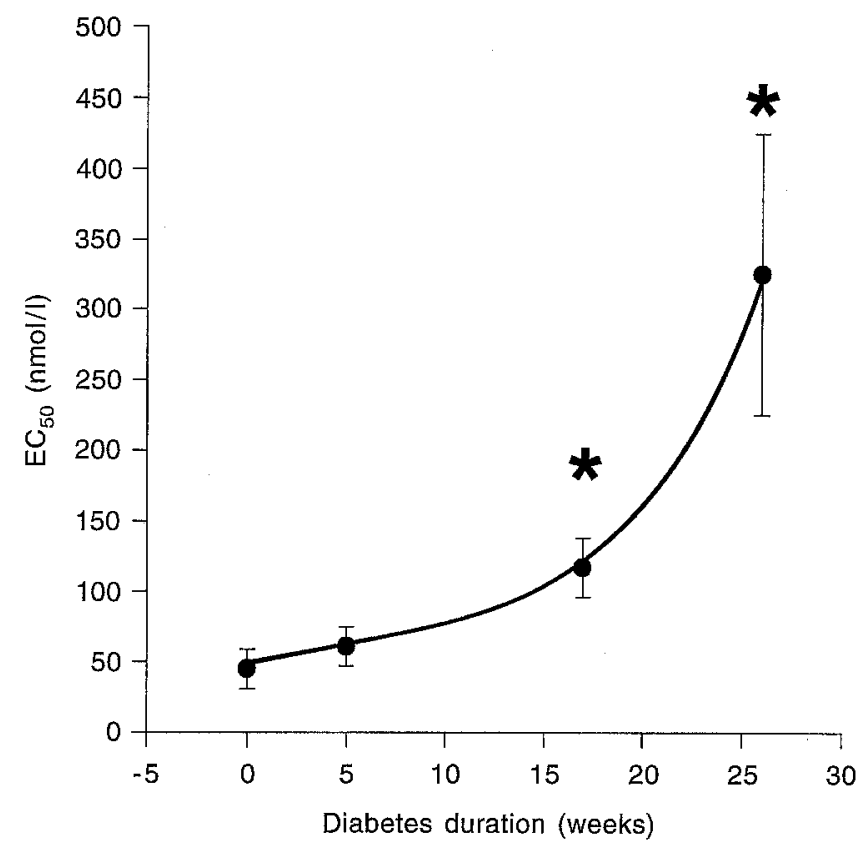

Fig.3. Influence of the diabetes duration on the sensitivity to 5-HT. The coronary flow in hearts of control and diabetic rats was determined depending on the concentration of 5-HT (cumulative dose-efficacy curve) and the duration of diabetes ranging from 5 to 26 weeks. Data are given as mean $\pm \mathrm{SEM}$ of six independent experiments. Significant differences between the various groups $* p<0.05$

Table 3. Concentration of 5-HT for half-maximal increase of coronary flow $\left(\mathrm{EC}_{50}\right)$

\begin{tabular}{lllc}
\hline Group & $\begin{array}{l}\text { Age } \\
\text { (weeks) }\end{array}$ & $\begin{array}{l}\text { Diabetes } \\
\text { (weeks) }\end{array}$ & $\begin{array}{l}\mathrm{EC}_{50} \\
(\mathrm{nmol} / \mathrm{l})\end{array}$ \\
\hline A: Control & 15 & - & $45 \pm 14$ \\
B: Control & 26 & - & $26 \pm 7$ \\
C: Diabetes & 15 & 5 & $61 \pm 31$ \\
D: Diabetes & 26 & 17 & $117 \pm 21^{\mathrm{a}}$ \\
E: Diabetes & 35 & 26 & $325 \pm 101^{\mathrm{a}}$ \\
F: + SOD & 26 & 17 & $59 \pm 15^{\mathrm{b}}$ \\
G: + Vitamin E & 26 & 17 & $15 \pm 9^{\mathrm{b}}$ \\
H: - Vitamin E & 26 & 17 & $502 \pm 214^{\mathrm{b}}$ \\
\hline
\end{tabular}

The half-maximal concentrations calculated by curve-fitting using the minimal root-mean square method

${ }^{a} p<0.05$ control $(\mathrm{B})$ vs diabetes $(\mathrm{D}, \mathrm{E})$

${ }^{\mathrm{b}} p<0.05$ diabetes (D) vs treated diabetes $(\mathrm{F}, \mathrm{G}, \mathrm{H})$

superoxide dismutase (SOD $50 \mu \mathrm{l} / \mathrm{ml}$ ). In control rats, perfusion with SOD had no influence on the basal coronary flow, the sensitivity of coronary vasculature to 5-HT or the maximal coronary flow (data not shown). In diabetes, however, SOD normalized the impaired sensitivity of the coronary vessels to 5-HT so that the $\mathrm{EC}_{50}$ was no longer significantly different from that of the controls (Table 3 ). In the diabetic rats the maximal coronary flow was slightly reduced in the presence of SOD.

Pretreatment with tocopherol. The experiments with SOD suggested that the generation of superoxide an- 


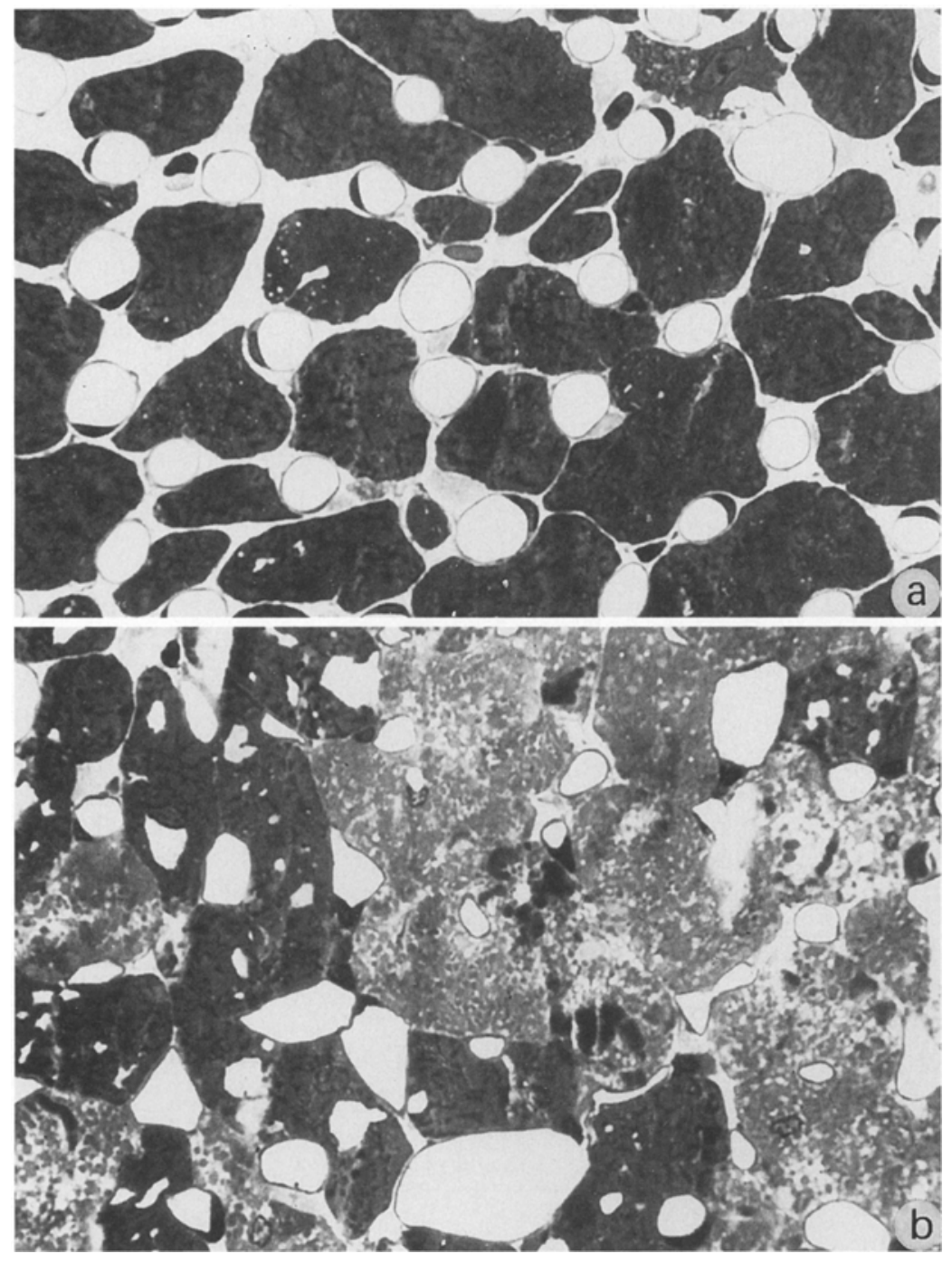

Fig.4 (a, b). Vitamin E protects myocardium in diabetic hearts. Samples processed as described in Methods. For light-microscopy ten semithin sections per heart were taken from the left papillary muscle of control $(n=5)$, diabetic $(n=5)$ and Vitamin E-treated, diabetic rats $(n=7)$ : a Myocardium of vitamin E-treated streptozotocin diabetic rats shows no sign of myocytolyses. The capillaries are evenly distributed throughout the tissue and show approximately the same diameters. b Untreated myocardium shows distinct focal necrosis and an irregular distribution and diameter of capillaries ions may be associated with the impairment of endothelium-dependent vasodilatation. To study the role of oxygen-derived radicals in the diabetic heart more closely, diabetic rats were treated with tocopherol-enriched and -deficient diets, respectively. The concentration of tocopherol in the plasma was significantly influenced by this treatment. Whereas a vitamin Edeficient diet diminished the plasma level of vitamin E from $8.2 \pm 0.4 \mathrm{mg} / \mathrm{dl}(0.19 \pm 0.009 \mathrm{mmol} / \mathrm{l})$ in controls to $2.3 \pm 0.2 \mathrm{mg} / \mathrm{dl}(0.05 \pm 0.005 \mathrm{mmol} / \mathrm{l})$, the vitamin E-enriched diet caused an increase up to $18.9 \pm 0.6 \mathrm{mg} / \mathrm{dl}(0.44 \pm 0.01 \mathrm{mmol} / \mathrm{l})$.

In control rats the tocopherol-modified diet has no influence on the coronary flow (data not shown). In diabetic rats coronary flow was, however, strictly altered by the pretreatment with the vitamin E-modified diets. Pretreatment with the vitamin E-enriched diet reduced the $\mathrm{EC}_{50}$ for the increase in coronary flow by $5-\mathrm{HT}$ to $15 \pm 9 \mathrm{nmol} / 1$ so that the sensitivity for 5-HT was not different in hearts of control and diabetic rats. Vitamin E deficiency, on the other hand, resulted in a further impairment of 5-HT mediated relaxation of coronary arteries. In the vitamin E-deficient state the $\mathrm{EC}_{50}$ was about 20-30-fold higher than in the hearts of control and diabetic rats treated with high doses of tocopherol (Table 3).

In parallel to the increased sensitivity to 5-HT, the structure of the myocardium was better preserved in the hearts of diabetic rats treated with high doses of tocopherol than in non-treated diabetic controls: typically, diabetes causes severe alterations of myocardial structure as shown (Figs. 4-6): myofilament bundles are deteriorated and partly fragmented. Areas of focal necrosis and contraction bands can be found at regular intervals. The number of perinuclear vacuoles is usually increased. Mitochondria are partly severely damaged and increased in diameter. Pretreatment with high doses of tocopherol resulted in an improved preservation of ventricular myocardium. The cardiac cells contained parallel arrangements of myofilaments and electron-dense mitochondria. The numbers of areas of focal necrosis and contraction bands as well as of intracellular vacuoles were clearly diminished in hearts of vitamin E-treated rats as 

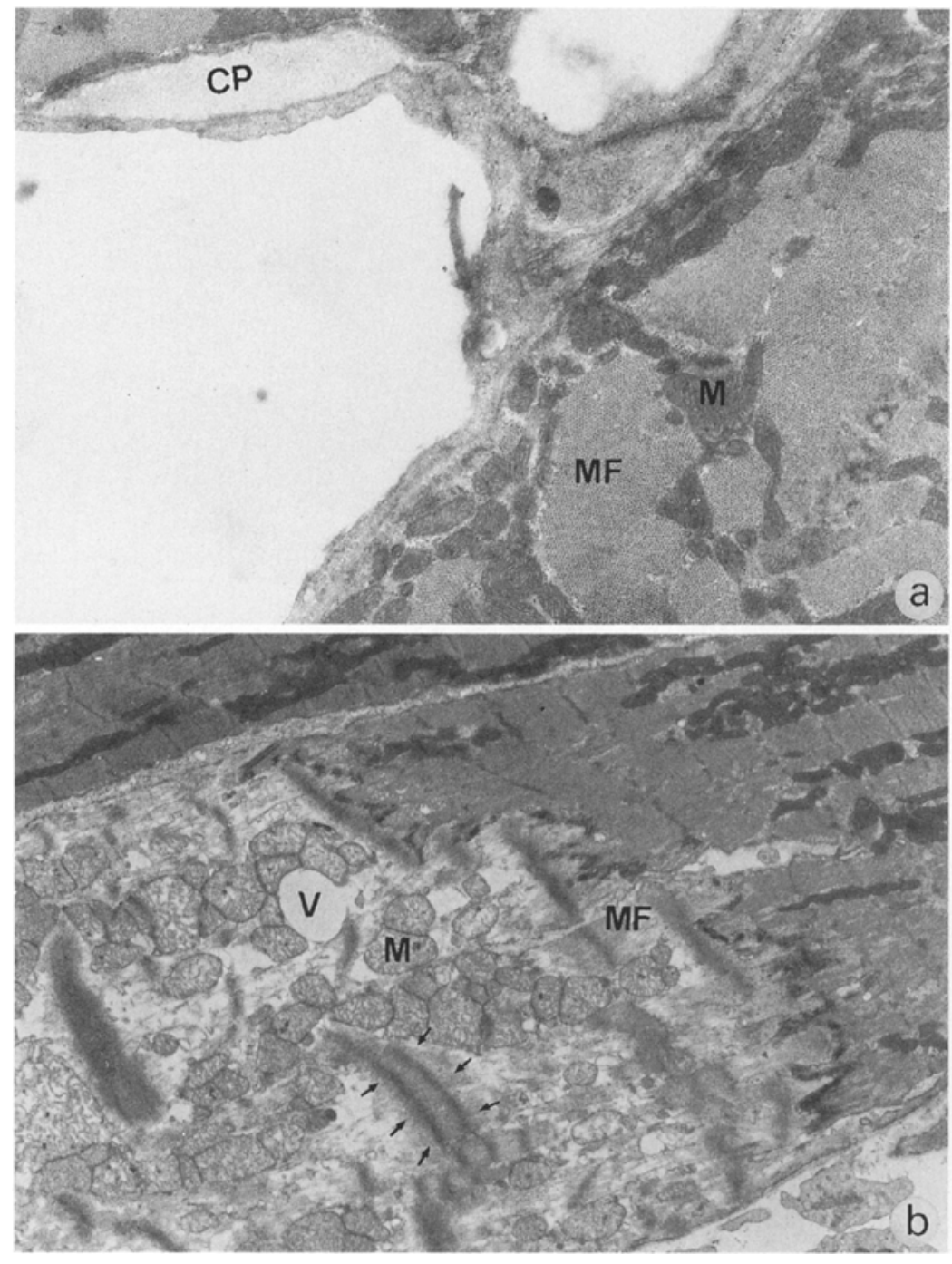

Fig.5 (a, b) Myocardium of diabetic rats. Samples processed as described in Methods. For electronmicroscopy five thin sections per heart were taken from the left papillary muscle of control $(n=5)$, diabetic $(n=5)$ and vitamin E-treated, diabetic rats $(n=7)$ :

a Transverse section of vitamin E-treated heart shows regular structure of myofilament bundles $(\mathrm{MF})$, regular size of mitochondria(M) and no intracellular vacuoles. There is only a small collagen plaque (CP) between capillary endothelial cell and myocyte.

b Longitudinal section of myocardium from un treated heart shows myocytes with varying stages of destruction. Swelling of mitochondria (M), intracellular vacuoles (V) and contraction bands can be found. In necrotic myocytes the structure of myofilaments (MF) is irregular and myofilaments are very sparse

compared to non-treated or vitamin $\mathrm{E}$ deficient diabetic rats (Figs. 4, 5).

Catecholamine fluorescence. In diabetic hearts histochemical visualization and quantification of intraneuronal catecholamines revealed a progressive loss of histofluorescent nerve fibers in the myocardium of streptozotocin-diabetic rats similar to that recently described for hearts of spontaneously diabetic (BB) rats [4]. These data indicate a loss of sympathetic nerve fibers and severe structural abnormalities that are very similar to alterations observed in peripheral nerves [35] (Figs. 6, 7). Treatment of the diabetic rats with vitamin $\mathrm{E}$ completely prevented this diabetic-specific loss of sympathetic nerve fibers and intraneuronal catecholamines. As in healthy myocardium the autonomic nerve fibers were running in parallel and the intraneuronal amount of catecholamines was not different from that of healthy control rats.

Collagen. It is known and has recently been described [4] that the interstitium of diabetic rat hearts revealed restrictive alterations; deposits of collagen fibers were frequently observed at precapillary locations in diabetic hearts, but not in those of healthy rats. These collagen deposits consisted of up to three orthogonal layers of bundles of collagen fibers [4]. In agreement with these observations the amount of collagen as determined by trichrome staining was also doubled in hearts of streptozotocin-diabetic rats $(+216 \%$, Fig. 9). Treatment of diabetic rats with vitamin $E$ reduced the number and the size of deposits of collagen I and III as shown in Figure 9. Changes in the ratio of collagen I and III were observed in hearts of untreated diabetic rats, but not in those of vitamin E-treated rats.

\section{Discussion}

Major observations of this study indicate that the endothelium regulation of coronary flow is progressively impaired in diabetes and that the generation of oxygen-derived radicals plays a decisive role in im- 

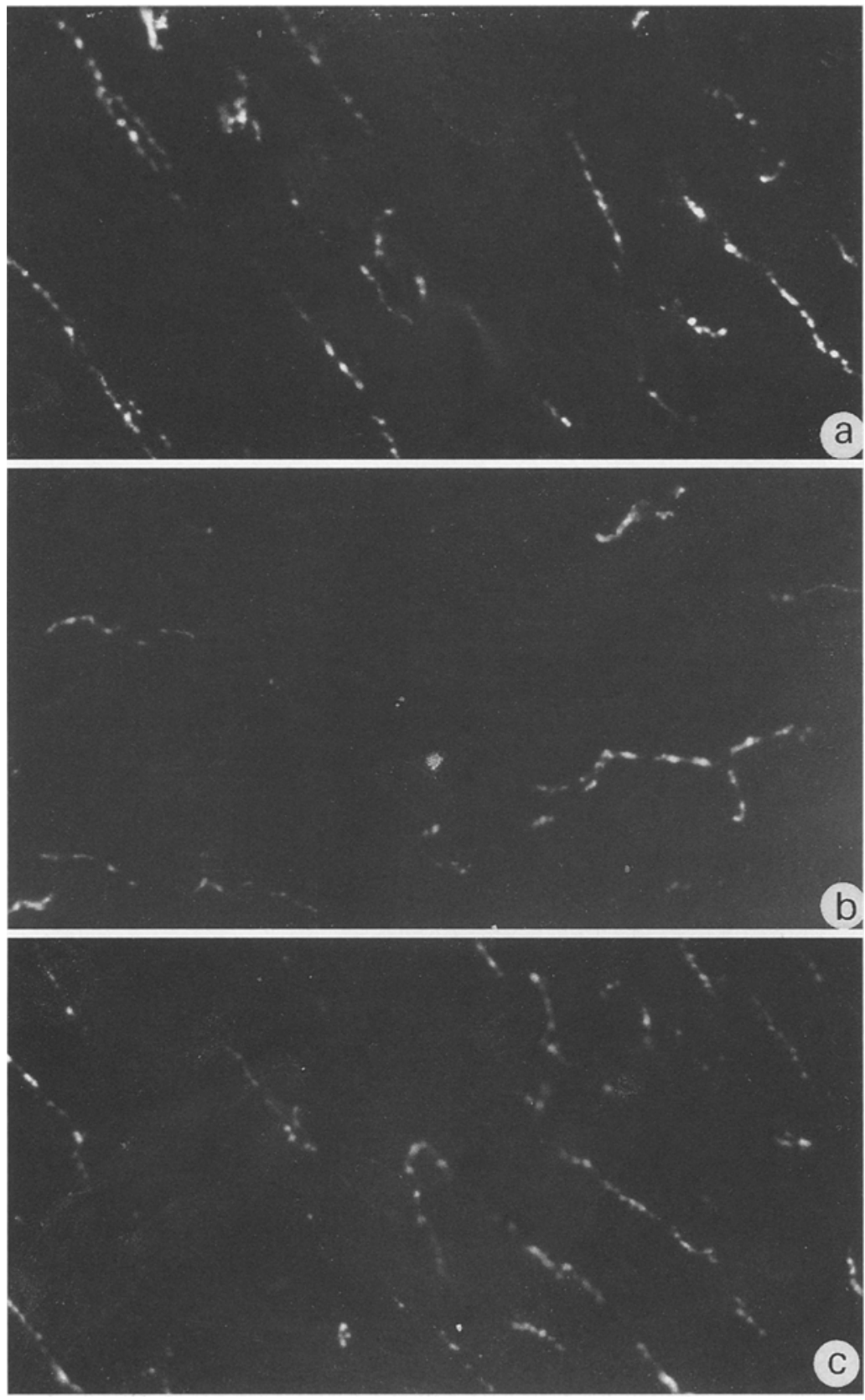

Fig. 6 (a-c). Autonomic neuropathy is prevented in vitamin E-treated diabetic heart. As described in Methods and by De La Torre \& Surgeon [32] the glyoxylic acid-induced fluorescence was determined in a section of the right ventricle taken in a distance of $150 \mu \mathrm{m}$ from the epicardial surface. Sections of control $(n=5)$, diabetic $(n=7)$ and vitamin E-treated, diabetic rats $(n=7)$ were studied: a In healthy myocardium fluorescent nerve fibres run parallel to the longitudinal axis of cardiomyocytes and form chain-like varicosities.

b Distinct reduction of fluorescent nerve fibers in untreated streptozotocin-diabetic rats.

c The disappearance of fluorescent nerve fibers is reduced paired endothelium-dependent vasodilatation. Pretreatment with tocopherol-acetate as anti-oxidant not only prevented this endothelial dysfunction, but also structural alterations of coronary vessels and depletion of myocardial catecholamine stores typically seen in the diabetic heart. These results strongly emphasize the pathophysiological relevance of oxygenderived radicals for endothelial and cardiac dysfunction in diabetes.

As criteria for the diabetic state induced by streptozotocin we used rats with blood glucose levels higher than $15 \mathrm{mmol} / 1$ and a level of $\mathrm{HbA}_{1}$ higher than $12 \%$. A significant difference in body weights between diabetic and control rats was only observed after a diabetes duration of at least 17 weeks, but not after 5 weeks of diabetes. These data indicate that the diabetic state induced by an intraperitoneal injection of $60 \mathrm{mg} / \mathrm{kg}$ body weight streptozotocin causes a distinct hyperglycaemia and a moderate inhibition of growth, but not an excessive catabolic state. The streptozotocin diabetes model used has already been extensively described by us and others $[4,7,26,31,36]$.

The heart preparation and the influence of diabetes has already been described extensively $[4,7,26$, $31,36]$. This standardized model was used to study the influence of diabetes on the endothelium-dependent regulation of coronary flow. To study specifically the effect of diabetes on the release of EDRF, the release of possibly interfering prostaglandins was inhibited by perfusion of the hearts in the presence 


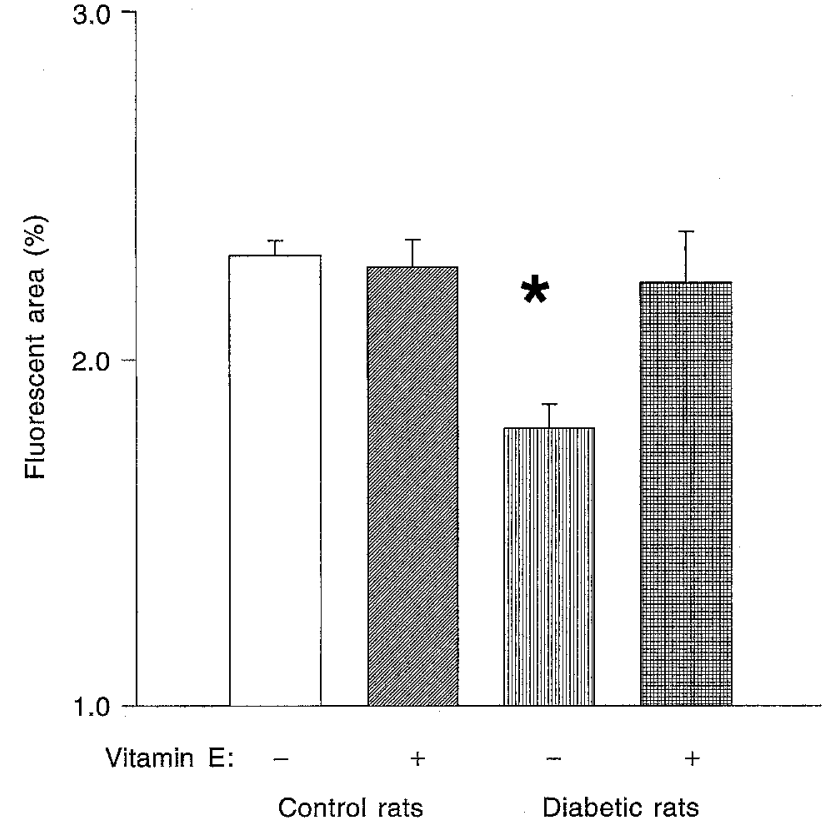

Fig. 7. Changes in catecholamine-related fluorescence. As described in Methods and by De La Torre [32] the glyoxylic acid induced fluorescence was determined by microfluorimetry. In a total area of $3 \mathrm{~mm}^{2}$ per heart the fluorescent area was measured by 100 consecutive measurements using a section of the right ventricle taken at a distance of $150 \mu \mathrm{m}$ from the epicardial surface. The fluorescent area is given as percentage of the total area: mean \pm SEM of control $(n=5)$, diabetic $(n=7)$, vitamin E-treated, diabetic rats $(n=7)$. $* p<0.05$ control as compared to diabetic rats

of indomethacin, which had no direct effect on the coronary flow in the concentration used [37]. Such inhibition of the release of prostaglandins is important, since it has already been shown that the myocardial release of cyclooxygenase products and particularly of prostacyclin is altered in the diabetic rat heart [5, 38]. Thus, in this particular in vitro model interference from prostaglandins can be excluded.

To stimulate the release of NO we used 5-HT instead of acetylcholine since it had been shown by others that acetylcholine does not only affect the coronary flow, but has additionally negative chronotropic and inotropic effects on the heart [39, unpublished results]. For 5-HT it has been shown previously that it specifically enhances the coronary flow without affecting myocardial contractility [40]. 5-HT led to a dose-dependent increase in coronary flow in hearts of control and diabetic rats, however, the sensitivity of the coronary vessels to 5-HT was progressively reduced in diabetes. Since neither the basal nor the maximal coronary flow capacity were impaired by diabetes, our data indicate that specifically the NO-mediated effect of 5-HT on the coronary vasculature is impaired in diabetes. Such an impairment of endothelium-dependent vasodilatation has been reported for various types of large (conductive) vessels [16, 17, 41]. Our data show that specifically the 5-HT stimula-
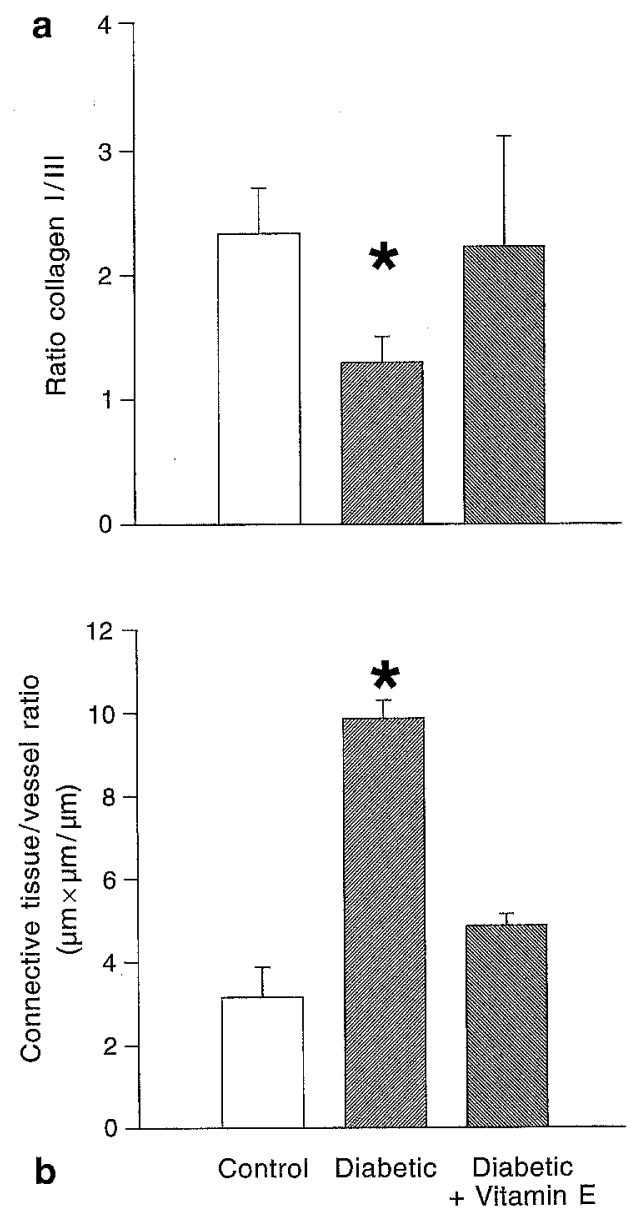

Fig. 8 (a, b). Effects of vitamin $E$ on perivascular connective tissue in diabetic hearts. The connective tissue was stained by the method described by Goldener [33] in consecutive slices from five different parts of the left coronary artery per heart: a Collagen I and III were determined by immuno-histochemical techniques as described in the Methods. The ratio of collagen I to III is given. $\mathbf{b}$ The amount of interstitial fibrosis was determined by the triochrome technique as described by Goldener [39] and in the Methods. The amount of trichrome-stained material is related to the vessel circumference. The ratio between the trichrome stained area (c in $\mu \mathrm{m}^{2}$ ) covering the vessel perimeter ( $\mathrm{v}$ in $\mu \mathrm{m}$ ) is given as mean $\pm \mathrm{SEM}$ of control $(n=5)$, diabetic $(n=7)$ and vitamin E-treated, diabetic rats $(n=7)$. The data are given as mean \pm SEM of control $(n=5)$, diabetic $(n=7)$ and vitamin E-treated, diabetic rats $(n=7)$. ${ }^{*} p<0.05$ control as compared to diabetic rats

tion of endothelium-dependent vasodilatation is disturbed in coronary resistance vessels of diabetic rats and that this endothelial effect becomes worse depending on the duration of diabetes. For the mesenteric bed similar observations have been reported by Diederich et al.[42] using acetylcholine as agonist. A recent study of Nitenberg et al. [25] suggests a similar impairment of vasodilatation in the large conduit vessels of the heart of IDDM and NIDDM patients again in response to acetylcholine. Since a comparable impairment in endothelium-dependent vasodilatation has been observed for various agonists (ace- 
tylcholine, bradykinin) and not only for 5-HT, we do not believe that this endothelial dysfunction is related to defects in the 5-HT receptor or in the signal transduction pathway, but by a defect further downstream.

The impairment of endothelium-dependent vasodilatation by the coronary bed could be abolished by perfusion with of SOD and prevented by tocopherol. Therefore, our observations suggest that the impairment of endothelium-dependent vasodilatation is presumably not a consequence of changes in the expression of the endothelium NO-synthase or in the sensitivity of the vascular smooth muscle cells for $\mathrm{NO}$, but that the heart in diabetes permanently generates superoxide anions which interfere with the NO-mediated vasodilatation. Such a mechanism for impairment of vasomotion in diabetes has already been postulated by others $[20,43,44]$. Direct evidence that free radicals and superoxide anions can mediate endothelial dysfunction and quench NO has already been presented [42-45]. It is assumed that oxygen free radicals affect the vascular systems by reacting with released NO to produce peroxynitrite which can decay to nitrogen dioxide and hydroxyradicals $[45,46]$. That the generation of free radicals is enhanced in diabetes is supported by considerable evidence [47, 48]. The source of these oxygenderived radicals is unclear and has not been identified by this study; the autoxidation of glucose $[49,50]$, lipid peroxidation $[47,48]$ as well as oxidation of glycated proteins [21] might lead to an increased release of oxygen-derived radicals and thereby exert an increased oxidative stress on the vasculature in diabetes. We can exclude, however, in contrast to studies using large vessels $[23,24]$ that prostaglandin endoperoxides or other cyclooxygenase products are involved in the impairment of endothelial function of coronary arteries, since the cyclooxygenase activity was completely inhibited by indomethacin.

As already outlined, it is intriguing to ascertain if the improvement of coronary perfusion and prevention of excessive release of catecholamines by NO are important mechanisms of cardioprotection in diabetes. Following this assumption pretreatment with tocopherol not only restored the endothelial-dependent vasodilatation in diabetic hearts, but also prevented the depletion of sympathetic nerve fibers from catecholamines (Figs. 7, 8), the deterioration of myocardial structure as well as the perivascular deposition of collagen and other matrix proteins.

We have to admit, however, that increased oxidative stress may not only affect the release of NO, but can lead to a variety of dysfunctions in diabetes [47]. In line with this assumption it has been shown very recently that tocopherol prevents the hyperglycaemia-induced activation of protein kinase $C$ [51] which has been suggested to represent a critical step in the development of vascular complications in diabetes [overview 14, 51]. Furthermore, there is much evidence that antioxidants inhibit the activation of platelets and monocytes in diabetic patients and restore the synthesis of prostacyclin [47]. Very recently, Kunisaki et al. [51] reported additionally that tocopherol prevents the glucose-induced biochemical changes in cultivated smooth muscle cells, but is also effective in vivo. Anti-oxidants may, therefore, be beneficial in various aspects of prevention of vascular, haemostatic and neurological complications in diabetes $[47,52]$. Thus, in addition to the restoration of NO release and its effects on the release of myocardial catecholamines other mechanisms might contribute to the cardioprotective effect of tocopherol.

Our observations do not exclude an increased formation of $\mathrm{NO}$ at very early states of hyperglycaemia as discussed by the "hyperfusion theory" [15]. They are relevant for diabetic states manifested for a prolonged time. The earliest changes in the regulation of coronary flow were observed after a diabetes duration of 5 weeks and might represent a long-term adaptation. We can exclude, on the other hand, that the observed changes in coronary perfusion are caused by changes in glucose concentration in the perfusion medium as well as by osmotic imbalances, since variation of glucose $(5-44 \mathrm{mmol} / \mathrm{l})$ or the addition of mannitol $(20-44 \mathrm{mmol} / \mathrm{l})$ did not affect the flow of the perfused rat heart (Rösen et al., unpublished results) in contrast to studies using vessels from other species [24].

In summary, our results suggest that oxidative stress plays a significant role in the development of endothelial dysfunction and the disturbances of cardiac structure and function in diabetes. Anti-oxidants such as tocopherol are able to prevent these adverse effects in vivo despite the elevated blood glucose levels and the increased formation of advanced glycation endproducts.

Acknowledgements. This work was supported by the Ministerium für Frauen, Familie und Gesundheit der Bundesrepublik Deutschland and the Wissenschaftsministerium des Landes NRW, the Deutsche Forschungsgemeinschaft, Bonn, and the "Klinische Zellbioloogie und Biophysik" e. V., Diusseldorf.

\section{References}

1. Rösen P, Pogatsa G, Tschöpe D, Addicks K, Reinauer H (1992) Diabetische Kardiopathie: Pathophysiologische Konzepte und therapeutische Ansätze. Klin Wochenschr 69: $3-15$

2. Uusitupa MIJ, Mustonen JN, Airaksinen KE (1990) Diabetic heart muscle disease. Ann Med 22: 377-386

3. Zoneraich S (1988) Small vessel disease, coronary vasodilation reserve, and diabetic microangiopathy. Chest 94: 5-7

4. Rösen P, Kiesel U, Reinauer H, Boy C, Addicks K (1991) Cardiopathy in the spontaneously diabetic (BB)rat: evidence for microangiopathy and autonomic neuropathy in 
the diabetic heart. In: Nagano M, Dhalla NS (eds) The diabetic heart. Raven Press, New York, pp 145-157

5. Rösen P, Rösen R, Hohl C, Reinauer H, Klaus W (1994) Reduced transcoronary exchange and prostaglandin synthesis in diabetes rat heart. Am J Physiol 247:H563-H569

6. Bhimji S, Godin DV, McNeill JH (1985) Biochemical and functional changes in hearts from rabbits with diabetes. Diabetologia 28: 452-457

7. Rodrigues B, McNeill JH (1992) The diabetic heart: metabolic causes for the development of a cardiomyopathy. Cardiovase Res 26: 913-922

8. Cannon RO, Cunnion RE, Parillo JE et al. (1987) Dynamic limitation of coronary vasodilator reserve in patients with dilated cardiomyopathy and chest pain. J Am Coll Cardiol 10: $1190-200$

9. Furchgott R, Zawadski J (1980) The obligatory role of endothelial cells in the relaxation of arterial smooth muscle by acetylcholine. Nature (London) 288: 373-376

10. Bassenge $E$ (1985) The role of endothelium and the local control of vascular tone. Basic Res Cardiol 85: 475-486

11. Ludmer PL, Selwyn AP, Shook TL et al. (1986) Paradoxical vasoconstriction induced by acetylcholine in atherosclerotic coronary arteries. N Engl J Med 315: 1046-1051

12. Zeiher AM, Drexler H, Wollschläger $H$, Just $H$ (1991) Modulation of coronary vasomotor tone in humans: progressive dysfunction with different early stages of coronary atherosclerosis. Circulation 83: 391-401

13. Brush JE, Faxon DP, Salmon S, Jacobs AK, Ryan TJ (1992) Abnormal endothelium-dependent coronary vasomotion in hypertensive patients. J Am Coll Cardiol 19: 809-815

14. Cohen RA (1993) Dysfunction of vascular endothelium in diabetes mellitus. Circulation 87[Suppl 5]:67-76

15. Williamson JR, Chang K, Frangos M et al. (1993) Hyperglycemic pseudohypoxia and diabetic complications. Diabetes: 42 : $801-813$

16. Meraji S, Jayakoby L, Senaratne MP, Thompson AB, Kappagoda T (1987) Endothelium-dependent relaxation in aorta of BB rat. Diabetes 36: 978-81

17. Oyama Y, Kawasaki H, Hattori Y, Kanno M (1986) Attenuation of endothelium-dependent relaxation in aorta of diabetic rats. Eur J Pharmacol 131: 75-78

18. McVeigh GE, Brennan GM, Johnston GD et al. (1992) Impaired endothelium-dependent and independent vasodilation in patients with type 2 (non-insulin-dependent) diabetes mellitus. Diabetologia 35: 771-776

19. Elliott TG, Cockcroft JR, Groop PH, Viberti GC, Ritter JM (1993) Inhibition of nitric oxide synthesis in forearm vasculature of insulin-dependent patients - blunted vasoconstriction in patients with microalbuminuria. Clinical Science 85: 687-693

20. Pieper GM, Gross GJ (1988) Oxygen free radicals abolish endothelium-dependent relaxation in diabetic rat aorta. Am J Physiol 255:H825-H833

21. Bucala R, Tracey KJ, Cerami A (1991) Advanced glycosylation products quench nitric oxide and mediate defective endothelium-dependent vasodilatation in experimental diabetes. J Clin Invest 87: 432-438

22. Tesfamariam B, Cohen RA (1992) Free radicals mediate endothelial cell dysfunction caused by elevated glucose. Am J Physiol 263:H321-H326

23. Tesfamariam B, Jakubowski JA, Cohen RA (1989) Contraction of diabetic rabbit aorta caused by endothelium-derived PGH2-TxA2. Am J Physiol 257:H1327-H1333

24. Tesfamariam B, Brown ML, Deykin D, Cohen RA (1990) Elevated glucose promotes generation of endothelium-derived vasoconstrictor prostanoids in rabbit aorta. J Clin Invest 85: 929-932
25. Nitenberg A, Valensi P, Sachs R, Dali M, Aptecar E, Attali JR (1993) Impairment of coronary vascular reserve and ACh-induced coronary vasodilation in diabetic patients with angiographically normal coronary arteries and normal left ventricular systolic function. Diabetes 42: 10171025

26. Toda N, Okamura T (1991) Role of nitric oxide in neurally induced cerebroarterial relaxation. J Pharmacol Exp Ther 258: 1027-1032

27. Romano FD, Kopp, SJ, Daar JT, Smith CA (1994) The antiadrenergic effect of cyclopentyladenosine on myocardial contractility is reduced in vivo in diabetic rats. Can J Physiol Pharmacol 72: 1245-1251

28. Burton KP (1988) Evidence of direct toxic effects of free radicals on the myocardium. Free Radical Biol Med 4: 1524

29. Bhambi B, Eghbali M (1991) Effect of norepinephrine on myocardial collagen gene expression and response of cardiac fibroblasts after norepinephrine treatment. Am J Pathol 139: 1131-1142

30. Pelouch V, Dixon IMC, Golfman L, Beamish RE, Dhalla NS (1993) Role of extracellular matrix proteins in heart function. Mol Cell Biochem 129: 101-120

31. Rösen P, Windeck P, Zimmer HG, Frenzel H, Burrig KF, Reinauer H (1986) Myocardial performance and metabolism in non-ketotic, diabetic rat hearts: myocardial function and metabolism in vivo and in the isolated perfused heart under the influence of insulin and octanoate. Basic Res Cardiol 81: 620-635

32. De La Torre JC, Surgeon JW (1976) A methodilogical approach to rapid and sensitive monoamine histofluorescence using a modified glyoxylic acid technique. The SPGmethod. Histochemistry 49: 81-93

33. Goldener $\mathbf{J}$ (1938) A modification of the Mason triochrome technique for routine laboratory purpose. Am J Pathol 14: 237-243

34. Diplock AT (1983) The role of vitamin $\mathrm{E}$ in biological membranes. In: Biology of vitamin E. (Ciba Foundation Sym) Pittman, London 101: 45-55

35. Addicks K, Boy C, Rösen P (1993) Sympathetic autonomic neuropathy in the heart of the spontaneous diabetic BB rat. Annals of anatomy. Anat Anz 175: 253-257

36. Tahiliani AG, McNeill JH (1986) Diabetes-induced abnormalities in the myocardium. Life Sci 38: 959-974

37. Vane JR, Ferreira SH (1979) Antiinflammatory drugs. In: Vane JR, Ferreira SH (eds) Handbuch der ExpPharmakologie. Springer, Berlin 50 I/II, pp 211-235

38. Roth DM, Reibel DK, Lefer AM (1983) Vascular responsiveness and eicosanoid production in diabetic rats. Diabetologia 24: 372-376

39. Ogiwara Y, Furukawa Y, Takeda M, Chiba S (1987) Blocking effects of alinidine on negative chronotropic and inotropic responses to vagal stimulation and injected acetylcholine and carbachol in dogs. J Pharmacol Exp Ther 243: $1113-1120$

40. Mertens H, Ballhausen T, Hollweg HG, Kirkpatrick JC, Kammermeier H (1994) Alteration of vascular endothelium and endothelium smooth muscle interaction after carbogen gas perfusion of isolated rat and guinea pig heart. Bas Res Cardiol 89: 322-340

41. Mayhan WG (1992) Impairment of endothelium-dependent dilatation of the basilar artery during diabetes mellitus. Brain Res 580: 297-302

42. Diederich D, Skopec J, Diederich A, Dai Fu-Xiang (1994) Endothelial dysfunction in mesenteric resistance arteries of diabetic rats: role of free radicals. Am J Physiol 266:H1153-H1161 
43. Pieper GM, Langenstroer P, Gross GJ (1993) Hydroxyl radicals mediate injury to endothelium-dependent relaxation in diabetic rat. Mol Cell Biochem 122: 139-145

44. Langenstroer P, Pieper GM (1992) Regulation of spontaneous EDRF release in diabetic rat aorta by oxygen radicals. Am J Physiol 263:H257-H265

45. Gryglewski RJ, Palmer RMJ, Moncada S (1986) Superoxide anion is involved in the breakdown of endothelin-derived vascular relaxing factor. Nature 320:454-456

46. Beckman JS, Beckman TW, Chen J, Marshall PA, Freeman BA (1990) Apparent hydroxyl radical production by peroxynitrite: implications for endothelial injury from nitric oxide and superoxide. Proc Natl Acad Sci USA 87: 16201624

47. Rösen P, Tschöpe D (1991) Vitamin E and diabetes. Fat Sci Technol 93: 425-431

48. Lyons TJ (1991) Oxidised low density lipoproteins: a role in the pathogenesis of atherosclerosis in diabetes? Diabetes Med 8: 411-419
49. Wolff SP, Dean RT (1987) Glucose autoxidation and protein modification. The potential role of oxidative glycosylation in diabetes. Biochem J 245: 243-250

50. Hunt JV, Smith CT, Wolff SP (1990) Autoxidative glycosylation and possible involvement of peroxides and free radicals in LDL modification by glucose. Diabetes 39: 1420-1424

51. Kunisaki M, Bursell SE, Umeda F, Nawata H., King GL (1994) Normalization of diacylglycerol-protein kinase C activation by vitamin $\mathrm{E}$ in aorta of diabetic rats and cultured smooth muscle cells exposed to elevated glucose levels. Diabetes 43: 1372-1377

52. Cameron NE, Cotter MA, Archibald V, Dines KC, Maxfield EK (1994) Anti-oxidant and pro-oxidant effects on nerve conduction velocity, endoneurial blood flow and oxygen tension in non-diabetic and streptozotocin-diabetic rats. Diabetologia 37: 449-459 